\title{
Survival of patients with intermediate stage hepatocellular carcinoma treated with superselective transarterial chemoembolization using doxorubicin-loaded DC Bead under cone-beam computed tomography control
}

\author{
Peter Popovic ${ }^{1}$, Borut Stabuc², Rado Jansa², Manca Garbajs ${ }^{1}$ \\ ${ }^{1}$ Clinical Institute of Radiology, University Medical Centre Ljubljana, Ljubljana, Slovenia \\ ${ }^{2}$ Gastroenterology Department, University Medical Centre Ljubljana, Ljubljana, Slovenia
}

Radiol Oncol 2016; 50(4): 418-426.

Received 10 June 2015

Accepted 19 August 2015

Correspondence to: Assist. Prof. Peter Popovič, MD, PhD, Clinical Institute of Radiology University Medical Centre Ljubljana, Zaloška cesta 7, SI-1525 Ljubljana, Slovenia. Phone: +38615223415; Fax: +38615222497; E-mail: peter.popovic@gmail.com

Disclosure: Peter Popovič reports personal fees from Terumo, Philips and Bayer outside the submitted work. The other authors declare that they have no conflict of interest.

Background. The purpose of this retrospective study was to evaluate treatment response, adverse events and survival rates of patients with intermediate stage HCC treated with superselective doxorubicin-loaded DC Bead transarterial chemoembolization (DEBDOX) under cone beam computed tomography (CBCT) control.

Patients and methods. Between October 2010 and June 2012, 35 consecutive patients with intermediate stage HCC (32 male, 3 female; average age, $67.5 \pm 7.8$ years; 22 patients Child-Pugh class A, 8 class B, 5 without cirrhosis) were treated with DEBDOX TACE. Portal vein thrombosis was observed in 6 (17.1\%) patients. DEBDOX TACE was performed by superselective catheterization of feeding vessels followed by embolization with 100-300 $\mu \mathrm{m}$ microspheres loaded with 50-100 mg of doxorubicin. In all cases, CBCT was used during chemoembolization. Tumor response rates were defined according to mRECIST criteria.

Results. Overall, 120 procedures were performed (mean, 3.2 per patients). We treated 97 lesions with an average diameter of $4.9 \pm 1.9 \mathrm{~cm}$. There were 32 minor and $2(1.6 \%)$ major complications (one liver abscess and one cerebrovascular insult). After a mean follow-up of $27.7 \pm 10.5$ months, $94.3 \%$ of patients achieved an objective response to treatment ( $42.4 \%$ complete response and $57.6 \%$ partial response). Mean time to progression was $10.9 \pm 5.3$ months. Mean overall survival was 33.9 months $(95 \% \mathrm{Cl} ; 28.9$ - 38.9 months), with 1- and 2-year survival of $97.1 \%$ and $65.7 \%$, respectively.

Conclusions. Superselective DEBDOX TACE performed under CBCT control is a safe and effective method with high rates of tumor response and overall survival.

Key words: hepatocellular carcinoma; chemoembolization; doxorubicin; drug eluting bead; cone-beam computed tomography

\section{Introduction}

Hepatocellular carcinoma (HCC) is the fifth most common cancer and the third most common cause of cancer mortality in the world. ${ }^{1}$ According to the Barcelona Clinic Liver Cancer (BCLC) staging sys- tem, curative therapies (resection, transplantation, and percutaneous ablation) can improve survival in HCC patients diagnosed at an early stage and offer potential long-term curative effects. ${ }^{1}$ About $20 \%$ of HCC patients are classified as BCLB-B, or intermediate-stage HCC. ${ }^{1}$ Transarterial chemoembolisation 
(TACE) is the standard treatment for patients with intermediate-stage HCC, but due to heterogeneity of the patient population in this stage, tumor response and survival rates are variable and scattered across the literature. ${ }^{2-4}$ Several clinical studies have confirmed the benefits of doxorubicin-loaded DC Bead (DEBDOX; drug-eluting bead doxorubicin] with respect to improved tumor response, reduced adverse events and improved survival. ${ }^{5-10}$ Furthermore, recent data shows that superselective TACE is associated with lower adverse events, higher rate of tumor response and increased survival. ${ }^{11-13}$ In superselective TACE, proper identification of the tumor feeding arteries and detection of the target tumor is crucial. ${ }^{11,12,14}$ However, angiography frequently cannot identify HCC lesions because of their small size or decreased hypervascularity. This often results in lobar or segmental TACE of relatively large liver areas, and in turn to lower rate of tumor response and potential increase in adverse events due to high repetition of TACE. Cone-beam CT (CBCT) is a novel technique that is increasingly used during TACE for inoperable HCC. ${ }^{15-20}$ This imaging technique uses a flat-panel detector angiographic system to produce CT-like soft-tissue images without the need to transfer the patient to a CT unit. ${ }^{15,17,19}$ It can be used to visualize tumor-feeding vessels and parenchymal stain during TACE, achieving detection accuracies significantly superior to standard 2D angiography. ${ }^{17}$ In this respect, the advantage of using CBCT during TACE improves the safety and effectiveness of the procedure, and has been suggested to have a benefit on local recurrence rates and overall survival during conventional TACE. ${ }^{20,21}$

The purpose of this retrospective study was to evaluate treatment response, adverse events and survival rates of patients with intermediate stage HCC treated with superselective DEBDOX TACE under $\mathrm{CBCT}$ control.

\section{Materials and methods}

\section{Patient selection}

This single institution study included 35 patients with intermediate stage HCC that were treated with DEBDOX TACE under CBCT control between October 2010 and June 2012. Clinical examination, laboratory evaluation and CT and/or MR imaging were performed in each patient at baseline at least one month before the TACE session. The inclusion criteria for DEBDOX-TACE were intermediate stage HCC according to BCLC system. Portal vein thrombosis was not an exclusion criterion.
Written informed consent of patients was obtained before the treatments. Ethics committee approval for treatment was provided for data analysis. Ethics committee approval for treatment was not required because TACE is approved as a standard of care for intermediate stage HCC. All the procedures followed the Helsinki declaration.

\section{TACE technique}

Treatment with chemoembolization was based on the consensus of the Liver Multidisciplinary Team Meeting, held weekly at our institution. All patients underwent at least two sessions of DEBDOX TACE. After local anesthesia, a short $5 \mathrm{~F}$ introducer sheath (Terumo Europe N.V., Belgium) was put in place via the common femoral artery. Digital subtraction angiography (DSA) of the celiac and superior mesenteric arteries was routinely performed via a 5 F catheter (Sidewinder ${ }^{\circledR}$, Terumo Europe N.V.) to determine vascular anatomy and variants and to assess portal flow. DSA was performed with the administration of non-ionic contrast agent iopromide $370 \mathrm{mg} / \mathrm{ml}$ (Ultravist $370 ®$, Bayer HealthCare, Germany) through a power injector (Avanta ${ }^{\circledR}$, Medrad, Bayer HealthCare).

The injection rate was normally $5 \mathrm{~mL} / \mathrm{s}$ with a total of $20 \mathrm{~mL}$ injected. A $2.4 \mathrm{~F}$ microcatheter (Progreat ${ }^{\circledR}$, Terumo Europe N.V.) was then superselectively positioned in the tumor feeding arteries before delivery of the DC Bead (DEBs) (DC Bead ${ }^{\circledR}$, Terumo Europe N.V.). Prior to DEBs delivery, DSA and CBCT were performed with the administration of iopromide through a power injector to confirm complete coverage of the targeted lesion(s). DEBs with a diameter of 100-300 $\mu \mathrm{m}$ were loaded with $50 \mathrm{mg}$ of doxorubicin per vial (maximum dose of $100 \mathrm{mg}$ of doxorubicin). In patients with multifocal tumors, the position of the microcatheter was changed within the same session to ensure superselective DEBs delivery in each lesion (Figure 1). Delivery of the mixture was continued until a near stasis end point or the antegrade blood flow was achieved. After DEBDOX TACE, completion arteriography was performed by manual injection to minimize reflux into non-targeted areas. All patients were kept under observation for a period of 24-48 h. Second DEBDOX TACE was performed after a period of 4-6 weeks in all patients. Additional chemoembolization procedures were performed if the multifocality of the disease did not allow for complete targeting of the tumor in the first two treatment sessions (Figure 2). DEBDOX TACE treatment was repeated on demand, that is, in pa- 

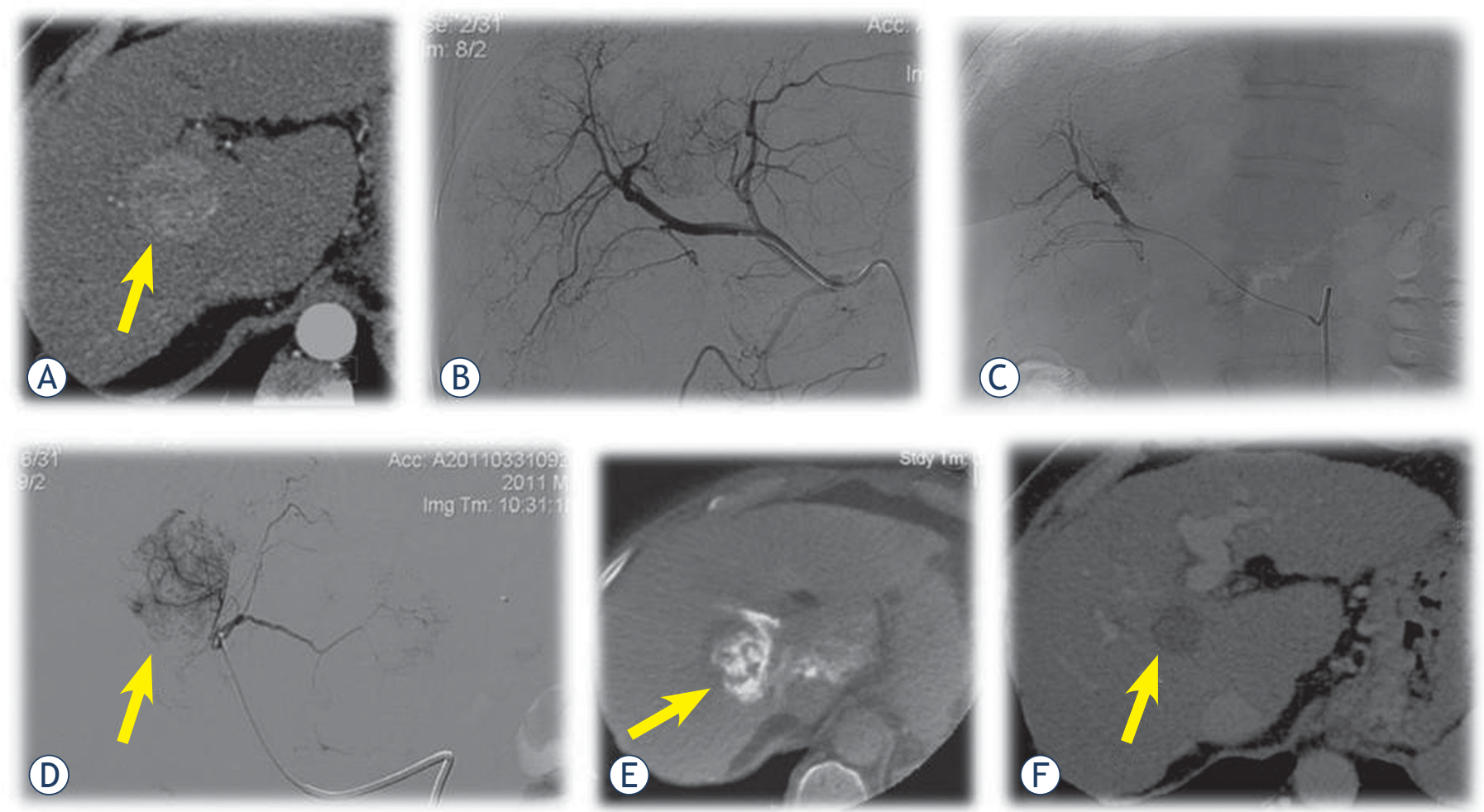

FIGURE 1. A 56-year-old female with HCC. (A) Contrast-enhanced CT shows tumor (arrow) between right and left liver lobe. (B) Initial angiography shows tumor in the liver (arrow). (C) Superselective contrast injection throught microcatheter into segmental branches for eight liver segment confirmes the tumor. (D) Superselective contrast injection throught microcatheter into segmental branches for first liver segment also confirmes the tumor (arrow). (E) CBCT after contrast injection throught microcatheter into segmental branches for first liver segment confirmes that the target lesion (arrow) is dominantely suplied from this artery and superselective DEBDOX TACE was performed from both artery. (F) Two months after the DEBDOX TACE, control CT shows complete devascularization of the target lesion (arrow) (complete response).

tients with residual or recurrent tumors observed by CT or MRI, according to the modified Response Evaluation Criteria in Solid Tumors (mRECIST) and in agreement with recent expert opinions. ${ }^{22}$

\section{Cone beam CT technique}

All patients underwent $\mathrm{C}$-arm single phase $\mathrm{CBCT}$. Imaging was performed by using a commercially available angiography system (Allura Xper FD20®; Philips HealthCare). This system was equipped with the XperCT option, enabling C-arm CBCT acquisition and volumetric image reconstruction (Feldkamp back projection). For each CBCT scan, the area of interest was positioned in the system isocenter, and, over approximately 10 seconds, 310 projection images were acquired with the motorized C-arm, covering a $240^{\circ}$ clockwise arc at a rotation speed of $20^{\circ}$ per second. As the images were being acquired, the projections were transferred via fiber-optic connection to the workstation (Philips Xtravision, Rel 6.2; Dell Precision 670; Round Rock, TX/USA). The two-dimensional projection images were reconstructed by using
Feldkamp back projection into three-dimensional volumetric images with isotropic resolution of 0.98 $\mathrm{mm}$ for a $250 \times 250 \times 194$-mm field of view (matrix size, $256 \times 256 \times 198)$. Typically, a $2.4-F r e n c h$ microcatheter (Progreat ${ }^{\circledR}$, Terumo Europe N.V.) was advanced into either a subsegmental or a segmental hepatic artery, depending on the location of the targeted tumor. CBCT was performed with the administration of non-ionic contrast agent (Ultravist $370 \circledR$, Bayer HealthCare) through a power injector (Avanta ${ }^{\circledR}$, Medrad, Bayer HealthCare). The injection rate was typically $1 \mathrm{~mL} / \mathrm{s}$ with a total injected volume of contrast agent $10 \mathrm{~mL}$ and delay time 15 seconds. The patients were instructed to be at endexpiration apnea during each CBCT acquisition. The final position of the microcatheter for delivery of the DEBs was based on the results of C-arm CBCT. This was done to superselectively deliver the bead to the tumors (Figure 3 ).

\section{Follow-up}

Follow-up after DEBDOX TACE included clinical evaluation, laboratory data, tumor treatment re- 
sponse and serious adverse events registration. Treatment response was evaluated with contrast enhanced four-phase computed tomography (CT) or magnetic resonance (MR) imaging with hepatospecific contrast media 2 months after DEBDOX TACE according to mRECIST. ${ }^{22} \mathrm{~A}$ viable tumor was defined by contrast agent uptake in the arterial phase and washout in the portal phase and/or late phase. Radiological follow up was performed every 3 months with either contrast enhanced four-phase CT or MRI with hepatospecific contrast media. CT was performed on a 64-row multidetector CT scanner (Siemens Medical Systems ${ }^{\circledR}$, Erlangen, Germany) and 16-row multidetector CT scanner (Siemens Medical Systems ${ }^{\circledR}$ ) with a fourphase protocol (non-enhanced, arterial, portal and late venous phase), which involved the administration of 100 to $130 \mathrm{ml}$ of non-ionic contrast agents (Ultravist 370@, Bayer HealtCare; Visipaque 320®, GE Healthcare) at a rate of $5 \mathrm{ml} / \mathrm{s}$ via a power injector by using a bolus tracking algorithm. Images were reconstructed at 5- and 2-mm thickness in axial and coronal planes.

MR imaging was performed on $3 \mathrm{~T}$ MAGNETOM Trio, A Tim Sistem scanner (Siemens Medical Systems ${ }^{\circledR}$ ). All images were acquired with a phased array abdominal coil. Routine protocol included imaging by using $\mathrm{T} 2$ weighted fast spin echo sequences, $\mathrm{T} 1$ weighted fat suppressed in- and out- of phase GRE, diffusion weighted imaging and dynamic enhanced multiphasic breath-hold $\mathrm{T} 1$ imaging by using hepatospecific contrast agent (Primovist ${ }^{\circledR}, \mathrm{Gd}-$ GD-EOB-DTPA, Bayer Schering Pharma AG, Germany). Images were acquired before contrast material application and during the arterial, portal, equilibrium, delayed and hepatospecific phase (after 20 minutes or more); $0.2 \mathrm{mM} / \mathrm{kg}$ of contrast agent was injected intravenously at a rate of $1 \mathrm{ml} / \mathrm{s}$ followed by $20 \mathrm{ml}$ of saline.

\section{Tumor response, time to progression and survival assessment}

Tumor response, mean time to progression and overall survival (OS) were calculated according to a follow-up time defined as the number of months from first DEBDOX TACE to $12^{\text {th }}$ August 2014, last imaging control of the patient, last contact with the patient or patient's death. Treatment response was determined at every follow-up imaging control, according to mRECIST. ${ }^{22}$ Patients with complete response or partial response were classified as having an objective response to treatment.
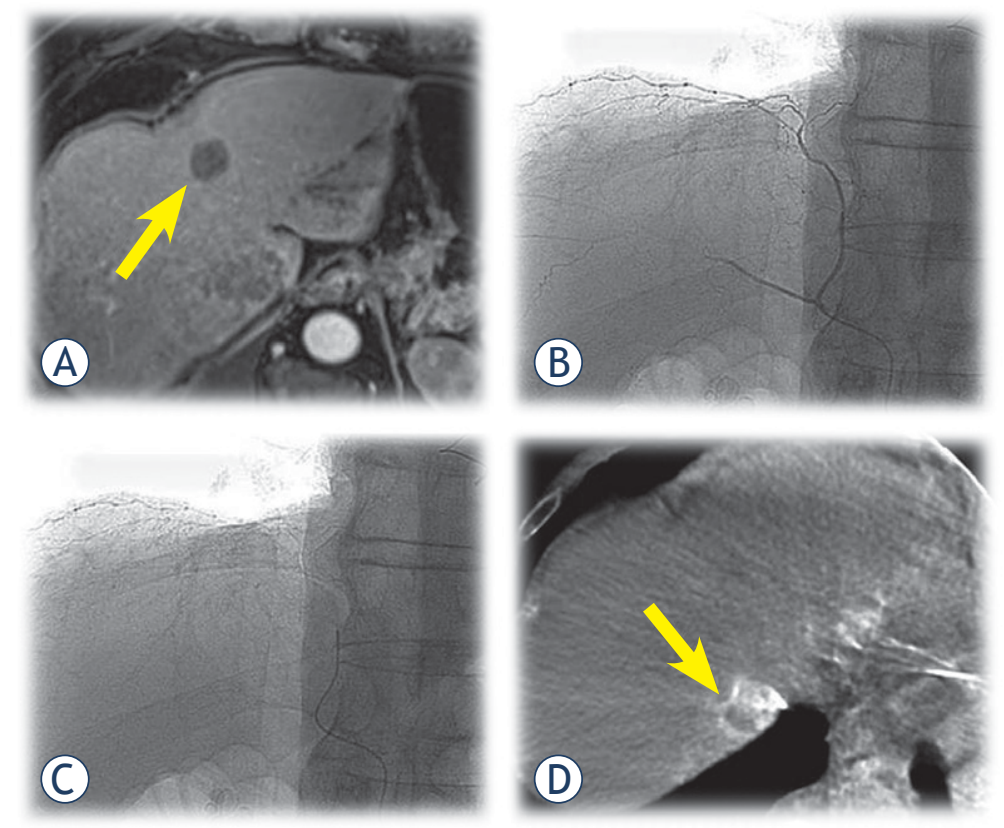

FIGURE 2. A 64-year-old male with HCC. (A) Control MR after chemoembolization shows complete response of the target lesion (arrow) in fourth segment and partial response of the target lesion in eight segment. (B) Superselective contrast injection throught microcatheter into the righr phrenic artery. (C) Position of microcatheter prior CBCT. (D) CBCT shows that this artery suplied the target tumor in seventh segment. Superselective DEBDOX TACE was performed.

\section{Statistical analysis}

Quantitative variables were expressed as mean \pm standard deviation and categorical as count and proportions. Survival rates and curves were determined using the Kaplan-Meier methods. Differences in the survival rate, regarding Child class were assessed using the log rank test. Last date for collection of data and calculating survival was $12^{\text {th }}$ August 2014. A $p$ value less than 0.05 was considered statistically significant.

Potential prognostic variables were evaluated as predictors of survival in the Cox proportional hazards model. All variables with a $p$ value $<0.05$ at univariate analyses were included in the multivariate analyses. All calculations were performed with statistical software (SPSS package version 19.0; SPSS Inc, Chicago, IL, USA).

\section{Results}

\section{Patient characteristics at baseline}

The baseline demographic, clinical, laboratory and tumor staging characteristics of the patients included in the analysis are summarized in Table 1. Mean 


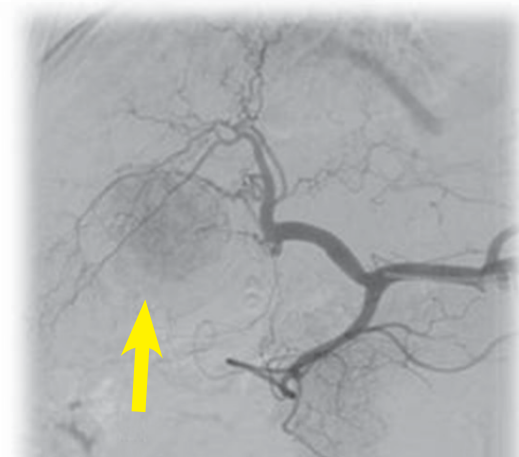

(A)

(D)
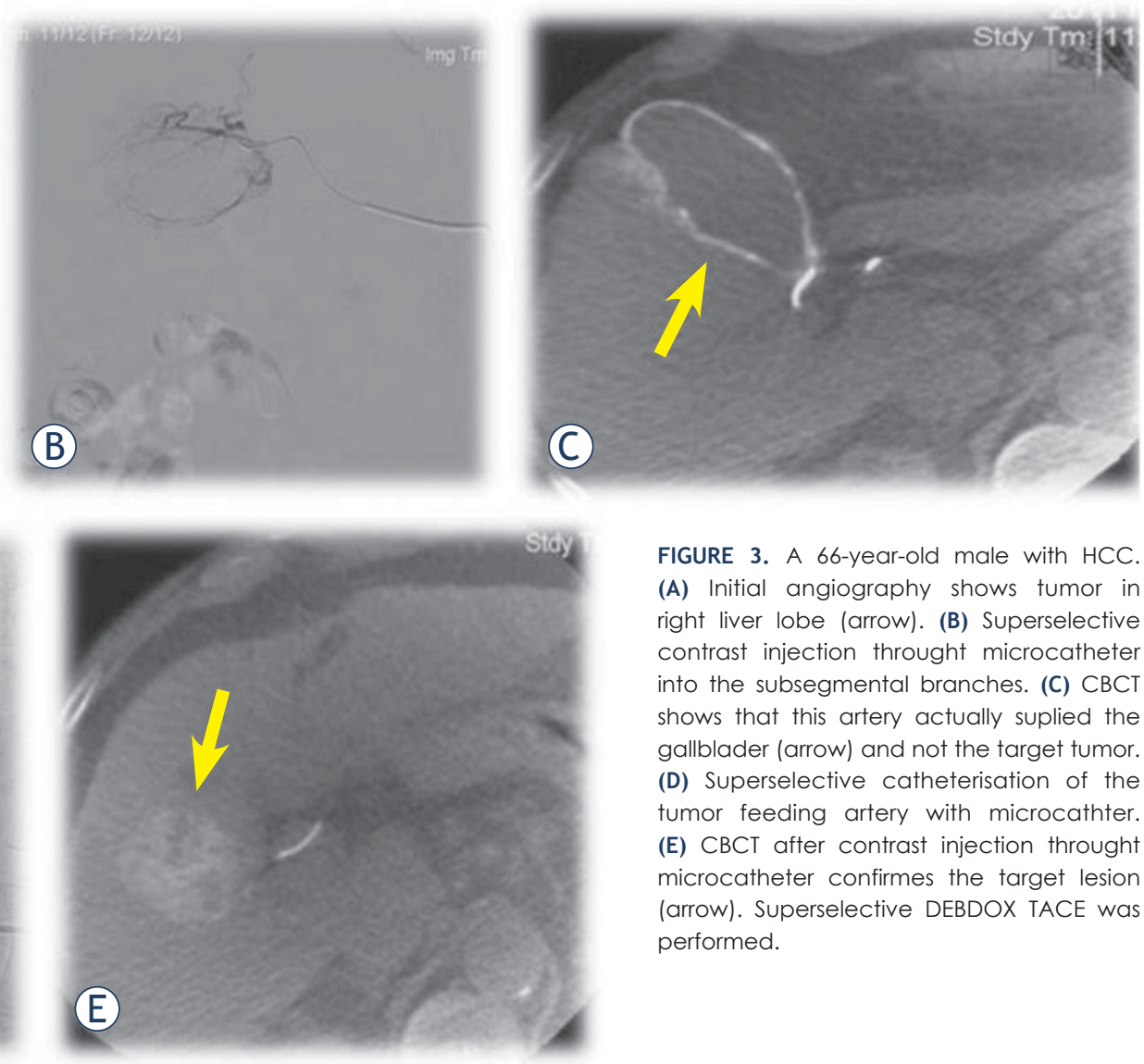

FIGURE 3. A 66-year-old male with HCC. (A) Initial angiography shows tumor in right liver lobe (arrow). (B) Superselective contrast injection throught microcatheter into the subsegmental branches. (C) CBCT shows that this artery actually suplied the gallblader (arrow) and not the target tumor. (D) Superselective catheterisation of the tumor feeding artery with microcathter. (E) CBCT after contrast injection throught microcatheter confirmes the target lesion (arrow). Superselective DEBDOX TACE was performed. patients age was $67.5 \pm 7.8$ years, the majority were male (32 of 35 patients). Five of 35 (11.4\%) patients were not cirrhotic. The most frequent etiology of cirrhosis was ethanol (43.3\%). Twenty-two patients were classified as Child-Pugh class A (73.3\%) and the remaining 8 as class B $(26.7 \%)$. Twenty-four of $35(68.6 \%)$ patients had unilobar, predominantly right lobe disease. Portal vein thrombosis was observed in $6(17.1 \%)$ patients.

\section{Procedure and complications}

Overall, 120 DEBDOX TACE procedures were performed in 35 patients. Mean number of procedures per patient was $3.2 \pm 1.5$. Superselective catheterization of feeding vessels was followed by embolization with 100-300 $\mu \mathrm{m}$ microspheres loaded with $50 \mathrm{mg}$ of doxorubicin. The maximum cumulative dose of doxorubicin was $100 \mathrm{mg}$. All procedures were performed under CBCT control.

Complications occurred in 34 of 120 procedures. There were two (1.6\%) major complications: an ischemic cerebrovascular insult to the cerebellum and an infection of the necrotic tumor that resolved after antibiotic treatment and resulted in prolonged hospitalization. Minor complications occurred in 32 procedures $(26.6 \%)$, of which 4 procedures resulted in two complications simultaneously, which were, however, etiologically different (Table 2).

\section{Response rate and time to progression}

Thirty-three of 35 patients (94.3\%) achieved an objective response after two sessions of DEBDOX TACE. Fourteen of 33 (42.4\%) patients achieved complete response, while $19(57.6 \%)$ achieved a partial response. Of the remaining two patients with no objective response, one patient had stable disease and the other had progressive disease (appearance of numerous new lesions in both liver lobes) despite continuous treatment with DEBDOX TACE. These patients were treated with sorafenib.

Twenty of 33 patients who had already achieved an objective response developed disease progression over time. Mean time to progression (TTP) was 10.9 months \pm 5.3 months (range, $5.8-24.8$ months). 


\section{Overall survival}

After a mean follow-up period of 27.7 months \pm 10.5 months, 17 patients died. One patient died of acute respiratory infection with acute respiratory insufficiency, two of spontaneous bacterial peritonitis with sepsis, one of respiratory arrest, two of toxic encephalopaty, four of progression of HCC and the remaining of liver failure. Mean OS of the whole cohort was 33.9 months (95\% CI: 28.9 - 38.9). 1-year survival was $97.1 \%$ and 2-year survival was $65.7 \%$. There was no significant difference in survival between patients with cirrhosis Child $\mathrm{A}$ and B $(p=0.417)$. Cox regression analysis showed that none of the clinical and laboratory data were statistically significant independent risk factors for survival.

\section{Discussion}

The purpose of this retrospective study was to evaluate treatment response, adverse events and survival rates of patients with intermediate stage HCC treated with superselective DEBDOX TACE under CBCT control. By facilitating tumor targeting and super-selective therapy delivery, our results support the hypothesis that CBCT plays a key role in achieving satisfactory tumor response and OS. 20,21

TACE is the most widely used loco-regional therapy for patients with intermediate stage HCC. The number of treatment sessions depends on the response of the tumor and whether serious side effects are seen..$^{3-5,7}$ The overall response rate for this treatment is about $50 \%$, with the lowest reported around $15 \%$ and the highest around $85.6 \% .^{2-5,16} \mathrm{In}$ a prospective study of 67 consecutive patients (122 nodules, all $<5 \mathrm{~cm}$ ), Golfieri et al. showed that, when compared with lobar conventional TACE (cTACE), selective/superselective CTACE was associated with higher mean levels of necrosis $(75.1 \%$ versus $52.8 \% ; p=0.002$ ) and a higher rate of complete necrosis $(53.8 \%$ versus $29.8 \%, p=0.013) .{ }^{11}$ These findings suggest that selective/superselective CTACE may determine a higher rate of tumor necrosis than lobar TACE. With improved treatment efficacy and tolerance, DEBDOX TACE represents a major advancement in treatment of intermediate stage HCC. $2,6,9,10,23$ In a recent prospective, randomized phase II study comparing CTACE with DEBDOX TACE, the DEBDOX TACE group showed a trend for a higher objective response rate than the CTACE group (51.6\% versus $43.5 \%$, respectively), together
TABLE 1. Baseline demographic, clinical, laboratory and tumor staging characteristics of patients

\begin{tabular}{|c|c|}
\hline Characteristic & Value \\
\hline Age, [years] & $67.5 \pm 7.8$ \\
\hline Gender (M/F), n [\%] & $32 / 3[91.4 / 8.6]$ \\
\hline Cirrhosis (yes/no), n [\%] & $\begin{array}{c}30 / 5[85.7 \\
/ 14.3]\end{array}$ \\
\hline $\begin{array}{l}\text { Etiology of cirrhosis, n [\%]: } \\
\text { Ethanol } \\
\text { HBV } \\
\text { HCV } \\
\text { other }\end{array}$ & $\begin{array}{ll}13 & {[43.3]} \\
4 & {[13.3]} \\
4 & {[13.3]} \\
9 & {[29.9]}\end{array}$ \\
\hline Albumin [g/l] & $38.4 \pm 4.6$ \\
\hline INR & $1.2 \pm 0.2$ \\
\hline Total bilirubin $[\mu \mathrm{mol} / \mathrm{l}]$ & $25.6 \pm 17.4$ \\
\hline Child-Pugh score (points) & $6.0 \pm 0.7$ \\
\hline $\begin{array}{l}\text { Child-Pugh score (classes), n [\%]: } \\
\text { A } \\
\text { B }\end{array}$ & $\begin{array}{l}22[73.3] \\
8[26.7]\end{array}$ \\
\hline Creatinine $[\mu \mathrm{mol} / \mathrm{l}]$ & $81.7 \pm 23.4$ \\
\hline ASAT $[\mu \mathrm{kat} / \mathrm{l}]$ & $1.1 \pm 0.7$ \\
\hline ALAT $[\mu \mathrm{kat} / \mathrm{l}]$ & $0.8 \pm 0.6$ \\
\hline YGT [ $\mu \mathrm{kat} / \mathrm{l}]$ & $2.3 \pm 1.6$ \\
\hline aFP $[\mathrm{kIE} / \mathrm{l}]$ & $152.5 \pm 310.2$ \\
\hline Portal vein thrombosis (yes/no), n [\%] & $6 / 29[17.1 / 82.9]$ \\
\hline Bilobar disease, $\mathrm{n}[\%]$ & $11[31.4]$ \\
\hline $\begin{array}{l}\text { Unilobar disease, n [\%:] } \\
\text { right lobe, } \mathrm{n}[\%]\end{array}$ & $\begin{array}{l}24[68.6] \\
20[83.3]\end{array}$ \\
\hline $\begin{array}{l}\text { Overall number of nodules, } n \\
\text { Number of nodules per pt, } n\end{array}$ & $\begin{array}{c}97 \\
2.8 \pm 2.2\end{array}$ \\
\hline Maximum diameter of $\mathrm{HCC}$ nodule per pt. [cm] & $4.7 \pm 1.9$ \\
\hline
\end{tabular}

$\mathrm{HBV}=$ hepatitis $\mathrm{B}$ virus, $\mathrm{HCV}=$ hepatitis $\mathrm{C}$ virus; $I N R=$ international normalised ratio; $A S A T=$ aspartate aminotransferase; $A L A T=$ alanine aminotransferase; $Y G T=$ gamma glutamyl transpeptidase; aFP = alpha-feto protein; $p t=$ patient. Quantitative variables expressed as mean \pm standard deviation.

with better tolerability. ${ }^{8,24}$ In another prospective, randomized study, DEBDOX TACE resulted in a better objective response than bland embolization with non-loaded bead $(73.1 \%$ versus $55.9 \%$, respectively). ${ }^{10}$ Similarly, Boatta and colleagues showed a $72 \%$ objective response rate in 154 patients treated with DEBDOX TACE. ${ }^{24}$ In a study by Suk et al., where CBCT after DEBDOX TACE was used to predict short-term tumor response according to mRECIST, objective response was achieved in $85.6 \%$ of patients (complete response $63.8 \%$, partial response $21.8 \%$ ), and objective response rates at one month after CBCT-guided TACE was also reported in a study by Loffroy et al. in $78 \%$ of patients (complete response $46.3 \%$, partial response $26.8 \%)^{2,16}$ In a retrospective study of 116 consecutive patients Georgiades et al. showed that delivery 
of two treatment sessions also lead to higher tumor response. ${ }^{25}$

Our results show a very high percentage $(91.9 \%)$ of objective response defined according to $\mathrm{mRE}$ CIST. The most plausible reasons for high objective response rate in our study were the routine utilization of CBCT control to guide superselective DEBDOX TACE in all patients and the delivery of at least two treatment sessions for all lesions.

TACE is also associated with OS benefits, reported in a meta-analysis of randomized controlled trials. ${ }^{3,26}$ Reported 1-, 2- and 3-year survival rates ranged from $24 \%$ to $90 \%, 59 \%$ to $81 \%$ and $29 \%$ to $47 \%$, respectively. $3,13,21,25,27$ However, most of these studies assessed cTACE. ${ }^{3,4,6}$ Studies reporting on survival of patients treated with DEBDOX TACE are scarce. A retrospective case-control study from a single academic institution in the United States demonstrated a survival benefit for patients undergoing DEBDOX TACE versus cTACE. ${ }^{23}$ One-year and 2-year survival were superior with DEBDOX TACE. ${ }^{28}$

In a recent study from Burrel et al. that analyzed strictly selected patients with intermediate stage HCC treated with DEBDOX TACE in a highly specialized center, reported 1- and 3 -year OS rates equal to $89.9 \%$ and $66.3 \%$, respectively. Median survival time in this study was 48.6 months. ${ }^{6}$ The overall favourable efficacy of DEBDOX TACE was further corroborated by a recent international, long-term (5 years of followup) study, where OS at 5 years was $22.5 \%$ and the mean OS was 43.8 months. ${ }^{29}$ To our knowledge there is only one study determining survival of HCC patients treated with TACE under CBCT control. This study showed that patients receiving CBCT-assisted CTACE had significantly higher OS rates than those receiving CTACE with angiography alone. OS rates of patients who underwent chemoembolization under CBCT assistance were $94 \%, 81 \%$ and $71 \%$ at 1,2 , and 3 years, respectively. ${ }^{21}$ OS rates in our study were $97 \%$ at 1 year, $66 \%$ at 2 years, with an average survival of 33.9 months. In particular, 1-year survival rate of our patients was higher than in most other studies reported in the literature, while 2-year survival was only marginally lower than the results reported by Iwazawa et al., where cTACE was also performed under CBCT control (2-year survival, 81\%). ${ }^{21}$ The high 1-year OS reported in our study may be related to the high objective response rates achievable with DEBDOX TACE, supported by the fact that objective response to treatment correlates with prolonged OS. ${ }^{30}$ In addition, the most com-
TABLE 2. Number and type of minor complications after DEBDOX TACE.

\begin{tabular}{lc}
\hline Type of complication & No. of complications \\
\hline Post-embolization syndrome* & 23 \\
Rise in blood pressure & 4 \\
Gastric erosions or ulcers & 2 \\
Chest pain & 2 \\
Hematoma at puncture site & 1 \\
\hline
\end{tabular}

*Post-embolization syndrome was defined as elevated body temperature pain in the abdomen, nausea and/or vomiting, leukocytosis and elevated pain in the abdor
liver enzymes.

mon reason for death in our study was not related to progression of HCC but rather to liver cirrhosis, which may explain the significant drop in 2-year survival.

TACE-associated adverse events, although usually transient and manageable, occur in a significant proportion $(35-100 \%)$ of patients, and may include post-embolization syndrome (comprising of fever, abdominal pain and a moderate degree of ileus), relevant liver function deterioration, ascites and gastrointestinal bleeding. ${ }^{11}$ Our analysis shows that DEBDOX TACE under CBCT control is a safe treatment method. The majority of complications were minor, either self limited or managed conservatively. One of the two major complications, infection of tumor necrosis, was due to the fact that necrotic tissue is an optimal culture medium for bacteria. ${ }^{6}$ The other major complication was a cerebrovascular insult. This was likely due to the reflux of the contrast and DEB particles mixture from an extrahepatic feeding artery (mammary artery) into the basilar artery that resulted in ischemic injury to the cerebellum. To our knowledge, 12 other cases with $25 \%$ mortality are reported in the literature. ${ }^{31}$ Our patient, however, died of liver failure 5 months after the procedure. Thus, no treatmentrelated deaths were reported.

We speculate that the utilization of intraprocedural CBCT and delivery of at least two treatment sessions for all lesions contributed to the high response rates, low occurrence of adverse events and good OS reported in our study. This imaging technique provides cross sectional, soft-tissue, CTlike images without the need to move the patient to a CT room and offers many advantages over classic 2D angiography alone. ${ }^{15,17,19,32}$ First, CBCT detection accuracy of HCC lesions is equivalent to multidetector CT and MRI and superior to 2D 
angiography. ${ }^{15,17,19}$ Second, it is the most accurate imaging technique to identify tumor-feeding arteries. ${ }^{14}$ Third, selective CBCT during hepatic angiography may be used to rule out non-target embolization to non-tumor feeding extrahepatic arteries and ensure that the selected branch supplies the tumor safety margin. ${ }^{17,18,33,34}$ In this respect, CBCT can also help with catheter placement when synchronous non-tumor and tumor enhancement are seen and can distinguish between tumor and non-tumor staining, most frequently occurring with feeding from the inferior phrenic artery. ${ }^{18}$ Finally, effective radiation doses for $\mathrm{CBCT}$ have been reported to be lower than with conventional. ${ }^{2,18}$

CBCT also has some limitations. Its contrast resolution is lower than that of conventional CT, thus providing less differentiation between HCCs and surrounding liver parenchyma. ${ }^{18,19}$ Furthermore, the detection of HCCs under diaphragm may be impaired by motion artefacts, while streak artefacts from the catheter and contrast material may also degrade image quality. ${ }^{15,18,19}$ The field of view of CBCT is smaller than that of conventional CT and liver truncation may occur. ${ }^{18}$ Finally, the acquisition of CBCT images requires additional contrast administration. This can be balanced with the use of CBCT guidance software to guide micro-catheter positioning to the targeted arteries, which may in turn help to reduce both radiation exposure and contrast administration. ${ }^{17}$

There are some limitations in our study. First, this study was a retrospective analysis and lacks a control group, thus failing to provide robust data of a prospective randomized controlled trial. In particular, we decided to avoid randomization between superselective DEBDOX TACE under CBCT and superselective DEBDOX TACE without CBCT, because of the anticipated clinical advantages of CBCT. Second, our small sample size may have introduced a selection bias. The BCLC B stage includes a heterogenous population of HCC patients and there is lack of a standard treatment methodology and patient selection criteria for TACE. ${ }^{25}$ Therefore, our patient selection criteria were strict in order to achieve the best outcome. Third, histopathologic correlation with CT or MR imaging regarding tumor necrosis after treatment with DEBDOX TACE was not performed since previous reports already showed good correlation between the percentage of tumor necrosis obtained at histopathologic examination and the tumor enhancement assessed with imaging. ${ }^{11,16}$

In conclusion, superselective DEBDOX TACE performed under CBCT control is a safe and effec- tive method with high tumor response and survival rates. Further prospective studies with a larger number of patients are required to elucidate the incremental benefit when compared to conventional techniques.

\section{References}

1. El-Serag HB, Rudolph KL. Hepatocellular carcinoma: epidemiology and molecular carcinogenesis. Gastroenterology 2007; 32: 2557-76.

2. Suk Oh J, Jong Chun H, Gil Choi B, Giu Lee H. Transarterial chemoembolization with drug-eluting bead in hepatocellular carcinoma: usefulness of contrast saturation features on cone-beam computed tomography imaging for predicting short-term tumor response. J Vasc Interv Radiol 2013; 24: 483-9.

3. Llovet JM, Bruix J. Systematic review of randomized trials for unresectable hepatocellular carcinoma: Chemoembolization improves survival. Hepatology 2003; 37: 429-42.

4. Lo CM, Ngan H, Tso WK, Liu CL, Lam CM, Poon RT, et al. Randomized controlled trial of transarterial lipiodol chemoembolization for unresectable hepatocellular carcinoma. Hepatology 2002; 35: 1164-71.

5. European Association For The Study Of The Liver and European Organisation For Research And Treatment Of Cancer. EASL-EORTC clinical practice guidelines: management of hepatocellular carcinoma. $J$ Hepatol 2012; 56: 908-43.

6. Burrel M, Reig M, Forner A, Barrufet M, de Lope CR, Tremosini S, et al. Survival of patients with hepatocellular carcinoma treated by transarterial chemoembolisation (TACE) using Drug Eluting Beads. Implications for clinical practice and trial design. J Hepatol 2012; 56: 1330-5.

7. Kim GM, Kim HC, Chung JW, Lee IJ, Kim HM, Jae HJ, et al. Chemoembolization for hepatocellular carcinoma supplied exclusively by the hepatic falciform artery. Cardiovasc Intervent Radiol 2012; 35: 845-51.

8. Lammer J, Malagari K, Vogl T, Pilleul F, Denys A, Watkinson A, et al. Prospective randomized study of doxorubicin-eluting-bead embolization in the treatment of hepatocellular carcinoma: results of the PRECISION V study. Cardiovasc Intervent Radiol 2010; 33: 41-52.

9. Lencioni R, Petruzzi P, Crocetti L. Chemoembolization of Hepatocellular Carcinoma. Semin Intervent Radiol 2013; 30: 3-11.

10. Malagari K, Pomoni M, Kelekis A, Pomoni A, Dourakis S, Spyridopoulos $\mathrm{T}$, et al. Prospective randomized comparison of chemoembolization with doxorubicin-eluting beads and bland embolization with BeadBlock for hepatocellular carcinoma. Cardiovasc Intervent Radiol 2010; 33: 541-51.

11. Golfieri R, Cappelli A, Cucchetti A, Piscaglia F, Carpenzano M, Peri E, et al. Efficacy of selective transarterial chemoembolization in inducing tumor necrosis in small $(<5 \mathrm{~cm})$ hepatocellular carcinomas. Hepatology 2011; 53: 1580-9.

12. Jin B, Wang D, Lewandowski RJ, Riaz A, Ryu RK, Sato KT, et al. Chemoembolization endpoints: effect on survival among patients with hepatocellular carcinoma. Am J Roentgenol 2011; 196: 919-28.

13. Takayasu K. Superselective transarterial chemoembolization for hepatocellular carcinoma: recent progression and perspective. Oncology 2011; 81(Suppl 1): 105-10.

14. Iwazawa J, Ohue $S$, Mitani $T$, Abe $H$, Hashimoto $N$, Hamuro $M$, et al. Identifying feeding arteries during TACE of hepatic tumors: comparison of C-arm CT and digital subtraction angiography. AJR Am J Roentgenol 2009; 192: 1057-63.

15. Higashihara $H$, Osuga $K$, Onishi $H$, Nakamoto A, Tsuboyama T, Maeda N, et al. Diagnostic accuracy of C-arm CT during selective transcatheter angiography for hepatocellular carcinoma: comparison with intravenous contrastenhanced, biphasic, dynamic MDCT. Eur Radiol 2012; 22: 872-9.

16. Loffroy R, Lin M, Yenokyan G, Rao PP, Bhagat N, Noordhoek N, et al. Intraprocedural C-arm dual-phase cone-beam CT: can it be used to predict short-term response to TACE with drug-eluting beads in patients with hepatocellular carcinoma? Radiology 2013; 266: 636-48. 
17. Floridi C, Radaelli A, Abi-Jaoudeh N, et al. C-arm cone-beam computed tomography in interventional oncology: technical aspects and clinical applications. Radiol Med 2014; 119: 521-32.

18. Tacher V, Radaelli A, Lin M, Geschwind JF. How I Do It: Cone-Beam CT during Transarterial Chemoembolization for Liver Cancer. Radiology 2015; 274: 320-34

19. Miyayama S, Yamashiro M, Okuda M, Yoshie $Y$, Sugimori N, Igarashi S, et al. Usefulness of cone-beam computed tomography during ultraselective transcatheter arterial chemoembolization for small hepatocellular carcinomas that cannot be demonstrated on angiography. Cardiovasc Intervent Radiol 2009; 32: 255-64.

20. Miyayama S, Yamashiro M, Hashimoto M, Hashimoto N, Ikuno M, Okumura $\mathrm{K}$, et al. Comparison of local control in transcatheter arterial chemoembolization of hepatocellular carcinoma $\leq 6 \mathrm{~cm}$ with or without intraprocedura monitoring of the embolized area using cone-beam computed tomography. Cardiovasc Intervent Radiol 2014; 37: 388-95.

21. Iwazawa J, Ohue S, Hashimoto N, Muramoto O, Mitani T. Survival after C-arm CT-assisted chemoembolization of unresectable hepatocellular carcinoma. Eur J Radiol 2012; 81: 3985-92.

22. Lencioni R, Llovet JM. Modified RECIST (mRECIST) assessment for hepatocellular carcinoma. Semin Liver Dis 2010; 30: 52-60.

23. Dhanasekaran R, Kooby DA, Staley CA, Kauh JS, Khanna V, Kim HS Comparison of conventional transarterial chemoembolization (TACE) and chemoembolization with doxorubicin drug eluting beads (DEB) for unresectable hepatocelluar carcinoma (HCC). J Surg Oncol 2010; 101: 476-80.

24. Boatta E, Corona M, Cannavale A, Fanelli F, Cirelli C, de Medici L. Endovascular treatment of hepatocellular carcinoma with drug eluting microparticles (DCBeads): CT evaluation of response to the treatment. Indian J Radiol Imaging 2013; 23: 126-33.

25. Georgiades C, Geschwind JF, Harrison N, Hines-Peralta A, Liapi E, Hong K, et al. Lack of response after initial chemoembolization for hepatocellula carcinoma: does it predict failure of subsequent treatment? Radiology 2012: 265: 115-23.

26. Lencioni R, Chen XP, Dagher L, Venook AP. Treatment of intermediate/ advanced hepatocellular carcinoma in the clinic: how can outcomes be improved? Oncologist 2010; 4(Suppl 15): 42-52.

27. Cabibbo G, Genco C, Di Marco V, Barbara M, Enea M, Parisi P, et al. Predicting survival in patients with hepatocellular carcinoma treated by transarterial chemoembolisation. Aliment Pharmacol Ther 2011; 34: 196204.

28. Huang $K$, Zhou $Q$, Wang $R$, Cheng $D$, Ma Y. Doxorubicin-eluting beads versus conventional transarterial chemoembolization for the treatment of hepatocellular carcinoma. J Gastroenterol Hepatol 2014; 29: 920-5.

29. Malagari K, Pomoni M, Moschouris H, Bouma E, Koskinas J, Stefaniotou A et al. Chemoembolization with doxorubicin-eluting beads for unresectable hepatocellular carcinoma: five-year survival analysis. Cardiovasc Intervent Radiol 2012; 35: 1119-28.

30. Kim BK, Kim SU, Kim KA, Chung YE, Kim MJ, Park MS, et al. Complete response at first chemoembolization is still the most robust predictor for favorable outcome in hepatocellular carcinoma. J Hepatol 2015; 62: 1304-10.

31. Zach V, Rapaport B, Yoo JY, Goldfeder L, Weinberger J. Multiple ischemic strokes after transcatheter arterial chemoembolization for hepatocellular carcinoma with a radiographic and pathological correlate. J Stroke Cerebrovasc Dis 2012; 21: 217-24.

32. Loffroy R, Favelier S, Cherblanc V, Estivalet L. C-arm dual-phase cone-beam $C T$ : a revolutionary real-time imaging modality to assess drug-eluting beads TACE success in liver cancer patients. Quant Imaging Med Surg 2013; 3: $196-9$.

33. Kim HC, Chung JW, Lee W, Jae HJ, Park JH. Recognizing extrahepatic collateral vessels that supply hepatocellular carcinoma to avoid complications of transcatheter arterial chemoembolization. Radiographics 2005; 1(Suppl 25): 25-39.

34. Miyayama S, Matsui O, Taki K, Minami T, Ryu Y, Ito C, et al. Extrahepatic blood supply to hepatocellular carcinoma: angiographic demonstration and transcatheter arterial chemoembolization. Cardiovasc Intervent Radio 2006; 29: 39-48. 